\title{
GROUP INVERSE OF FINITE POTENT ENDOMORPHISMS ON ARBITRARY VECTOR SPACES
}

\author{
FERnANDO PABLOS ROMO
}

Abstract. The aim of this work is to introduce the group inverse of a finite potent endomorphism on an infinite-dimensional vector space that generalizes the notion of group inverse of a square finite matrix. The existence and uniqueness of this inverse is proved, several properties are offered and the relations with Drazin inverse, CMP inverse and DMP inverses are studied.

Mathematics subject classification (2010): 15A09, 15A03, 15A04.

Keywords and phrases: Group inverse, Drazin inverse, CMP inverse, EP endomorphism, DMP inverse, finite potent endomorphism.

\section{REFERENCES}

[1] M. Argerami, F. Szechtman, R. Tifenbach, On Tate's trace, Linear Multilinear Algebra 55 (6), (2007) 515-520.

[2] V. Cabezas Sánchez, F. Pablos Romo, Moore-Penrose Inverse of Some Linear Maps on InfiniteDimensional Vector Spaces, Electron. J. Linear Algebra 36, (2020) 570-586.

[3] S. L. CAMPBelL, JR. MEYER, Generalized Inverses of Linear Transformations, Dover, (1991). ISBN 978-0-486-66693-8.

[4] S. Cheng, Y. TIAn,, Two sets of new characterizations for normal and EP matrices, Linear Alg. Appl. 375, (2003) 181-195.

[5] S. B. Malik, N. Thome, On a new generalized inverse for matrices of an arbitrary index, Appl Math Comput. 226 (1), (2014) 575-580.

[6] M. Mehdipour, A. SAlEmi, On a new generalized inverse of matrices, Linear and Multilinear Algebra 66 (5), (2018) 1046-1053, doi : 10.1080/03081087.2017.1336200.

[7] F. Pablos Romo, Classification of Finite Potent Endomorphisms, Linear Algebra and Its Applications 440, (2014) 266-277.

[8] F. Pablos Romo, Core-Nilpotent Decomposition and new generalized inverses of Finite Potent Endomorphisms, Linear and Multilinear Algebra 68 (11), (2020) 2254-2275.

[9] F. Pablos Romo, On Drazin-Moore-Penrose Inverses of Finite Potent Endomorphisms, Linear and Multilinear Algebra (2019), doi : 10.1080/03081087.2019.1612834.

[10] F. Pablos Romo, On the Drazin Inverse of Finite Potent Endomorphisms, Linear and Multilinear Algebra 67 (10), (2019) 2135-2146.

[11] J. TATE, Residues of Differentials on Curves, Ann. Scient. Éc. Norm. Sup. 1, 4a série, (1968) 149-159. 